\title{
Quantifying regional left ventricular contractile function: Leave it to the machines?
}

\author{
John J. Squiers, BSE, ${ }^{a}$ Mani Arsalan, MD, ${ }^{b, c}$ Jeffrey E. Thatcher, $\mathrm{PhD},{ }^{\mathrm{d}}$ and J. Michael DiMaio, MD ${ }^{\mathrm{a}, \mathrm{b}}$
}

See related article on pages $240-6$.

In this issue of the Journal, Henn and colleagues ${ }^{1}$ from Washington University in St Louis present a novel method to quantify and localize regional left ventricle (LV) contractile function in coronary artery disease by means of cardiac magnetic resonance imaging with radiofrequency tissue tagging. They have developed a mechanism by which the quantified LV function can be compared against a normalized standard to determine the presence and severity of an individual patient's pathologic contractile dysfunction. Henn and colleagues ${ }^{1}$ deserve praise for developing an automated, quantitative solution for a problem that has only been addressed qualitatively in the past. The ability to both quantify and localize LV contractile dysfunction should improve clinical outcomes in several ways by addressing limitations of the current nonquantitative metrics of regional LV function. First, this method creates an objective standard that will increase consistency and accuracy across the board by reducing the interuser and intertemporal variability that has plagued echocardiographic interpretation of LV function in the past. Furthermore, the simplicity of interpreting and displaying these results may increase patients' understanding of their coronary artery disease.

A key component of this study is the methodology to extrapolate a single measurement of cardiac wall motion ( $z$ score) from detailed and multidimensional data and to construct a reference range model by "normalizing" the LV function $z$ score of a healthy cohort, a process similar to what is known as machine learning in engineering circles. Machine learning typifies a ubiquitous engineering approach to solving complex biological questions-and its application is only increasing in frequency within the biomedical realm. ${ }^{2}$ Machine learning takes advantage of computer models that can be taught to perform a desired task. Training data are collected to develop and

\footnotetext{
From the ${ }^{\mathrm{a}}$ Department of Epidemiology, Baylor Healthcare System, Dallas, Tex; ${ }^{\mathrm{b}}$ The Heart Hospital Baylor Plano, Plano, Tex; ${ }^{\mathrm{c} D e p a r t m e n t ~ o f ~ C a r d i a c ~ S u r g e r y, ~ K e r c k h o f f ~}$ Clinic Heart Center, Bad Nauheim, Germany; and ${ }^{\mathrm{d}}$ Spectral MD, Inc, Dallas, Tex. Disclosures: Authors have nothing to disclose with regard to commercial support. Received for publication April 14, 2015; accepted for publication April 16, 2015; available ahead of print May 16, 2015.

Address for reprints: J. Michael DiMaio, MD, Baylor Healthcare System, 6125 Luther Ln, Dallas, TX 75225 (E-mail: jmdimaio@yahoo.com).

J Thorac Cardiovasc Surg 2015;150:247-9

$0022-5223 / \$ 36.00$

Copyright (c) 2015 by The American Association for Thoracic Surgery http://dx.doi.org/10.1016/j.jtcvs.2015.04.034
}

fine-tune a model that is subsequently implemented to classify new data. The model can be constructed and reconstructed with training algorithms in a recursive process to increase its classification accuracy. ${ }^{3}$ A summary of this process as applied by Henn and colleagues ${ }^{1}$ is presented in Figure 1.

The biomedical interest in machine learning derives from its potential to enhance the sensitivity and specificity of diagnosis while additionally increasing objectivity in physician decision-making. ${ }^{4}$ This technique has already been used in a surprisingly diverse set of applications, including automatic interpretation of electrocardiograms, ${ }^{5}$ diagnosis of pigmented skin lesions, ${ }^{6}$ and prediction of outcomes in patients with diffuse large B-cell lymphoma. ${ }^{7}$ We believe that machine learning has the potential to drastically change day-to-day clinical practice in virtually every field of medicine by improving physicians' diagnostic and treatment capabilities.

Henn and colleagues ${ }^{1}$ collected data from cardiac magnetic resonance imaging with radiofrequency tissue tagging in a healthy cohort to serve as training data, and the resulting model (a Gaussian reference range of normalized LV contractile function) was implemented to classify LV contractile function in patients with coronary artery disease. Henn and colleagues ${ }^{1}$ were able to develop a highly accurate model ( $\geq 89 \%$ ), as judged by established, qualitative markers for wall motion abnormalities (electrocardiography, single-photon emission computed tomography, and echocardiography). Again, it is important to emphasize that not only did this model prove to be accurate (ie, discriminating functional vs dysfunctional), but it also was able to provide quantitative data regarding the patients' LV function (ie, degree of dysfunction).

The diagnostic model developed by Henn and colleagues ${ }^{1}$ shows great promise, and their work leads to several additional questions from the machine learning perspective. Typically, the most costly part of model development in machine learning is in obtaining samples that represent the true-positive and true-negative populations. This aspect of development must be guided by experts in the respective medical field, because this part of the process is often the most scrutinized as the product is translated from benchtop experiments to real-world, clinical situations. Certainly, further investigation into the technology developed by Henn and colleagues ${ }^{1}$ will continue to require a strong focus on developing a model from the appropriate data.

The decision to report a $z$ score in a color-coded format allows easy interpretation of the results, which is an 


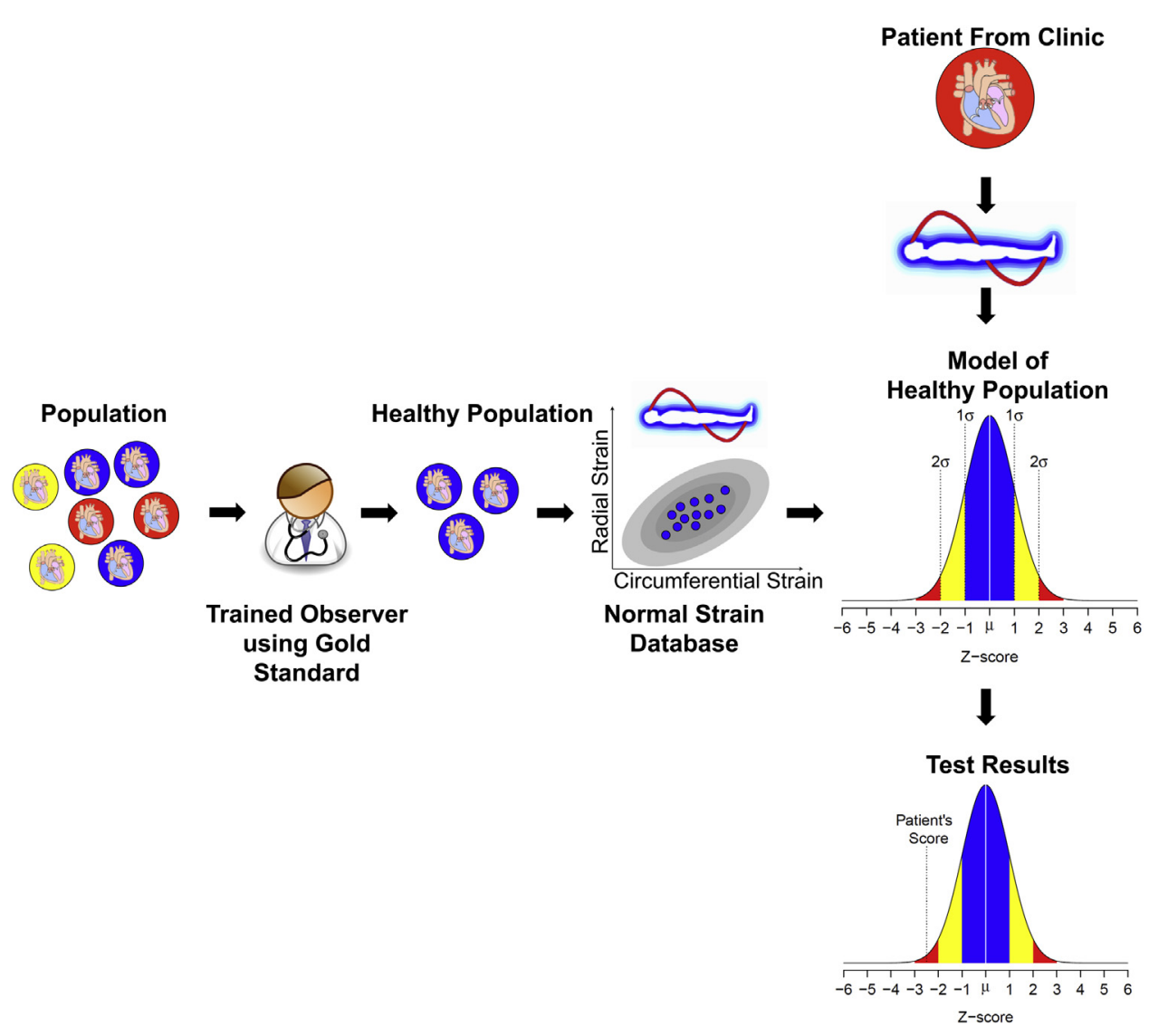

FIGURE 1. Computer algorithm approach to quantifying a patient's left ventricular function. First, development of the "normal strain" database is supervised by the current best clinical knowledge (horizontal arm). Patients that come into the clinic are then tested, and their results are compared with the normal distribution of cardiac strains (vertical arm). The categorization as diseased or not diseased and quantification of the dysfunction, if present, are determined by the patient's data fit to the database, which stresses the importance of acquiring the proper data to train the classification algorithm.

implicit recognition by Henn and colleagues ${ }^{1}$ that machine learning outputs must be immediately relatable to clinical practice, so that products may be more readily adopted by physicians. If the machine output is even a single step removed from a physical measure such as concentration, weight, electrocardiographic tracings, and so on, an explanation of the surrogate measure $(z$ score, in this case) must be intuitive or clearly available along with the output results. Quantitative results from machine learning algorithms are also more valuable when they are presented with a measure of confidence. As this technology is further developed, we hope to see such a measure reported.

Henn and colleagues ${ }^{1}$ have classified significant LV contractile dysfunction according to the traditional statistical analysis cutoff of $2 \mathrm{SD}$ - the same cutoff used in interpreting blood tests commonly ordered by physicians. More work is necessary to determine whether this cutoff is appropriate for clinical application in this particular instance. For example, can the proposed classification scheme accurately predict patient prognosis or determine the optimal revascularization strategy for each patient?
Finally, no computer algorithm can be guaranteed to work in $100 \%$ of situations. For this reason, it is essential that physicians be made aware of circumstances in which the use of the technology is contraindicated or significantly less likely to report accurate results. Such situations are the very reason that machine learning technology can never replace the physician. Just as electrocardiograms automatically read by the machine are overread and confirmed by a physician, the output of any computer algorithm must be considered in the clinical context by a physician capable of recognizing when the algorithm has erred.

We commend Henn and colleagues ${ }^{1}$ for developing an intuitive, quantitative method to classify LV contractile function that has the potential to spur significant improvement in clinical decision making relative to the inconsistent, qualitative tests used today. They are certainly on track to build a useful tool despite the high costs of magnetic resonance imaging, because their technology delivers results with excellent accuracy and without any influence of observer bias. Machine learning is sure to improve clinical practice in this and many other ways in the very near future. 
The authors thank Leah Gaither for her editorial assistance.

\section{References}

1. Henn MC, Cupps BP, Kar J, Kulshrestha K, Koerner D, Braverman AC, et al. Quantifying "normalized" regional left ventricular contractile function in ischemic coronary artery disease. J Thorac Cardiovasc Surg. 2015;150:240-6.

2. Foster KR, Koprowski R, Skufca JD. Machine learning, medical diagnosis, and biomedical engineering research-commentary. Biomed Eng Online. 2014;13:94.

3. Alpaydin E. Introduction to machine learning. 3rd ed. Boston: MIT Press; 2014.
4. Sajda P. Machine learning for detection and diagnosis of disease. Annu Rev Biomed Eng. 2006;8:537-65.

5. Polat K, Güneş S. Detection of ECG arrhythmia using a differential expert system approach based on principal component analysis and least square support vector machine. Appl Math Comput. 2007;186:898-906.

6. Dreiseitle S, Ohno-Machado L, Kittler H, Vintervo S, Billhardt H, Binder M. A comparisons of machine learning methods for the diagnosis of pigmented skin lesions. J Biomed Inform. 2001;34:28-36.

7. Shipp MA, Ross KN, Tamayo P, Weng AP, Kutok JL, Aguiar RC, et al. Diffuse large B-cell lymphoma outcome prediction by gene-expression profiling and supervised machine learning. Nat Med. 2002;8:68-74. 